\title{
Degree homogeneous subgroups
}

\author{
John D. Dixon* \\ Carleton University, Ottawa, Canada \\ A. Rahnamai Barghi \\ Institute of Advance Studies in Basic Sciences, Zanjan, Iran
}

September 29, 2004

\begin{abstract}
Let $G$ be a finite group and $H$ be a subgroup. We say that $H$ is degree homogeneous if, for each $\chi \in \operatorname{Irr}(G)$, all the irreducible constituents of the restriction $\chi_{H}$ have the same degrees. Subgroups which are either normal or abelian are obvious examples of degree homogeneous subgroups. Following a question of E.M. Zhmud', we investigate general properties of such subgroups. It appears unlikely that degree homogeneous subgroups can be characterized entirely by abstract group properties, but we provide mixed criteria (involving both group structure and character properties) which are both necessary and sufficient. For example, $H$ is degree homogeneous in $G$ if and only if the derived subgroup $H^{\prime}$ is normal in $G$ and, for every pair $\alpha, \beta$ of irreducible $G$-conjugate characters of $H^{\prime}$, all irreducible constituents of $\alpha^{H}$ and $\beta^{H}$ have the same degree.
\end{abstract}

Mathematics Subject Classification (2000): 20C15

*The first author acknowledges partial support from NSERC Operating Grant A7171. The second author's visit to Carleton University was made possible through this grant and he also thanks Carleton University for its hospitality during the time that this paper was written. 


\section{Introduction}

Let $G$ be a finite group and $H$ be a subgroup. We say that $H$ is degree homogeneous (and write $(G, H) \in \Delta$ ) if, for each $\chi \in \operatorname{Irr}(G)$, all the irreducible constituents of the restriction $\chi_{H}$ have the same degrees. We have two obvious examples of degree homogeneous subgroups: normal subgroups are degree homogenous by Clifford's theorem, and abelian subgroups are degree homogeneous because their irreducible characters are all of degree 1. Following a question in [2, page 304], our object is to investigate general properties of such pairs $(G, H)$. As Lemma 1 shows, it is unlikely that the class $\Delta$ can be characterized entirely by abstract group properties, but Theorems 5,12 and 9 give mixed criteria (involving both group structure and character properties) for these pairs. Theorems 17 and 18 describe some related classes.

Notation We shall generally follow the notation of [6]. In particular, $\operatorname{Irr}(G)$ denotes the set of all irreducible (ordinary) characters of $G, \operatorname{Lin}(G)$ is the set of characters of degree 1 and, for any character $\theta, \operatorname{Irr}(\theta)$ is the set of irreducible constituents of $\theta$. If $K$ is a normal subgroup of $G$, and $\alpha \in \operatorname{Irr}(K)$, then $I_{G}(\alpha):=\left\{x \in G \mid \alpha^{x}=\alpha\right\}$ is the inertia subgroup of $\alpha$. We use $c d(G)$ to denote the set of degrees of the irreducible characters of $G$.

\section{Degree homogeneous subgroups}

We begin with some simple properties of $\Delta$.

Lemma 1 (a) If $\left(G_{i}, H_{i}\right) \in \Delta$ for $i=1,2$ then $\left(G_{1} \times G_{2}, H_{1} \times H_{2}\right) \in \Delta$.

(b) If $(G, H) \in \Delta$ and $H \leq L \leq G$, then $(L, H) \in \Delta$.

(c) If $(G, H) \in \Delta$ and $K \leq H$ is normal in $G$, then $(G / K, H / K) \in \Delta$.

Proof. (a) Every irreducible character $\chi$ of $G_{1} \times G_{2}$ has the form $\chi((x, y))=$ $\theta(x) \phi(y)$ for all $(x, y) \in G_{1} \times G_{2}$ where $\theta \in \operatorname{Irr}\left(G_{1}\right)$ and $\phi \in \operatorname{Irr}\left(G_{2}\right)$, and conversely every function of this form is an irreducible character (see, for example, [6, (4.21)]). Thus the irreducible constituents of the restriction of $\chi$ to $H_{1} \times H_{2}$ are characters of the form $\psi((x, y))=\lambda(x) \mu(y)$ where $\lambda$ and $\mu$ are irreducible constituents of $\theta_{\mathrm{H}_{1}}$ and $\phi_{\mathrm{H}_{2}}$, respectively. The assertion now follows.

(b) By Frobenius reciprocity every irreducible character $\psi$ of $L$ is an irreducible constituent of some $\chi \in \operatorname{Irr}(G)$. Now (b) follows since $\operatorname{Irr}\left(\psi_{H}\right) \subseteq$ $\operatorname{Irr}\left(\chi_{H}\right)$.

(c) This follows at once since each irreducible character of $G / K$ corresponds to some $\chi \in \operatorname{Irr}(G)$ of the same degree with $K \leq \operatorname{ker} \chi$.

Suppose that $(G, H) \in \Delta$. Then for each $d \in c d(H)$ we define

$$
\mathcal{C}_{d}:=\left\{\chi \in \operatorname{Irr}(G) \mid \text { all constituents of } \chi_{H} \text { have degree } d\right\}
$$

and

$$
\mathcal{D}_{d}:=\{\theta \in \operatorname{Irr}(H) \mid \theta(1)=d\}
$$


Set

$$
K_{d}:=\bigcap_{\chi \in \mathcal{C}_{d}} \operatorname{ker} \chi \text { and } L_{d}:=\bigcap_{\theta \in \mathcal{D}_{d}} \operatorname{ker} \theta
$$

Then the following holds.

Theorem 2 If $(G, H) \in \Delta$ then, with the notation above, $K_{d}=L_{d}$, and so $L_{d} \triangleleft G$. In particular, $H^{\prime}=L_{1} \triangleleft G$ and $L_{d} \leq H^{\prime}$ whenever $d \in \operatorname{cd}(H)$

Proof. If $x \in L_{d}$, then clearly $\chi(x)=\chi(1)$ for all $\chi \in \mathcal{C}_{d}$, and so $x \in$ $K_{d}$. Conversely, by Frobenius reciprocity, $\theta \in \mathcal{D}_{d}$ if and only if $\operatorname{Irr}\left(\theta^{G}\right) \subseteq \mathcal{C}_{d}$. Thus $x \in K_{d}$ implies that $\theta^{G}(x)=\theta^{G}(1)$ for each $\theta \in \mathcal{D}_{d}$. Since $\operatorname{ker} \bar{\theta}^{G}=$ $\bigcap_{y \in G} y^{-1}(\operatorname{ker} \theta) y \leq \operatorname{ker} \theta$, we conclude that $x \in L_{d}$. Thus $K_{d}=L_{d}$ as asserted.

The group $L_{1}$ is simply the intersection of the kernels of the linear characters of $H$ and so $L_{1}=H^{\prime}$. Moreover, if $\theta \in \operatorname{Irr}(H)$ has degree $d$, then for each linear characters $\lambda$ of $H, \theta \lambda \in \operatorname{Irr}(H)$ and so $\theta \lambda \in \mathcal{D}_{d}$. Hence $x \in L_{d}$ implies that $\theta(x) \lambda(x)=\theta(x)=d$ for each linear character $\lambda$, and so $x \in \operatorname{ker} \lambda$. Thus $L_{d} \leq L_{1}$ as claimed.

As an immediate corollary we get:

Corollary 3 Suppose that $(G, H) \in \Delta$. If $G$ is simple and $H \neq G$, then $H$ must be abelian. On the other hand if $H$ is perfect, then $H$ must be normal.

In general, the necessary conditions given in Theorem 2 are not sufficient for a subgroup to be degree homogeneous as the following example shows.

Example 4 Let $V$ be the regular embedding in $S_{9}$ of the elementary abelian 3 -group of order $3^{2}$. Define $G$ as the normalizer of $V$ in $S_{9}$. Then $G$ is the holomorph of $V$, and a point stabilizer $L$ in $G$ is isomorphic to $G L(2,3)$. The group $L$ contains a single conjugacy class of elementary abelian 2-groups of order $2^{2}$; let $A$ be one of these groups. Finally define $H:=V A$.

A simple computation shows that $H^{\prime}=V \triangleleft G$. Using GAP [3] we find that $G$ has a unique irreducible character $\chi$ of degree 16 and that $\chi_{H}=\psi_{1}+\psi_{2}+\psi_{3}+$ $\psi_{4}+2 \phi$ where the irreducible constituents $\psi_{1}, \psi_{2}, \psi_{3}, \psi_{4}, \phi$ are of degrees $2,2,2,2$ and 4 , respectively. Hence $H$ is not degree homogeneous in $G$. GAP also shows that the characters of $H$ are all of degrees 1,2 or 4 and that $L_{2}=L_{4}=1$ (so all the necessary conditions of Theorem 2 are satisfied). Note also that $H$ is a product of two degree homogeneous subgroups, namely, the normal abelian subgroup $V$ and the abelian subgroup $A$.

(An alternative proof that $H$ is not degree homogeneous can be given using the theorem below.)

We can characterize the pairs which lie in $\Delta$ as follows.

Theorem 5 Let $H$ be a subgroup of a finite group $G$. If $(G, H) \in \Delta$, then for each normal subgroup $K$ of $G$ with $H^{\prime} \leq K \leq H$ we have

$\left(^{*}\right)$ if $\alpha, \beta \in \operatorname{Irr}(K)$ are $G$-conjugate, then all constituents of $\alpha^{H}$ and $\beta^{H}$ have the same degree 
(Recall that $H^{\prime} \triangleleft G$ by Theorem 2).

Conversely, if there exists at least one normal subgroup $K$ of $G$ with $H^{\prime} \leq$ $K \leq H$ such that $\left(^{*}\right)$ is satisfied, then $(G, H) \in \Delta$.

Proof. First recall (see [6, Problem (6.2)]) that, because $H / K$ is abelian, the irreducible constituents of $\alpha^{H}$ all have the same degree (and, of course, similarly for $\beta^{H}$ ).

Since $\alpha, \beta \in \operatorname{Irr}(K)$ are $G$-conjugate, therefore $\alpha^{G}=\beta^{G}$. Choose $\chi \in$ $\operatorname{Irr}\left(\alpha^{G}\right)$. By Frobenius reciprocity, $\alpha$ and $\beta$ are irreducible constituents of $\chi_{K}$. Thus for some $\psi, \phi \in \operatorname{Irr}\left(\chi_{H}\right)$ we have $\alpha \in \operatorname{Irr}\left(\psi_{K}\right)$ and $\beta \in \operatorname{Irr}\left(\phi_{K}\right)$. Equivalently, $\psi \in \operatorname{Irr}\left(\alpha^{H}\right)$ and $\phi \in \operatorname{Irr}\left(\beta^{H}\right)$.

If $(G, H) \in \Delta$, then $\psi(1)=\phi(1)$, and so all constituents of $\alpha^{H}$ and $\beta^{H}$ have the same degree by the remark at the beginning of this proof. Thus $(*)$ holds for every normal subgroup $K$ of $G$ with $H^{\prime} \leq K \leq H$. This proves the first part of the theorem.

Conversely, assume that ${ }^{*}$ ) holds for some normal subgroup $K$ of $G$ with $H^{\prime} \leq K \leq H$, and $\chi$ is an arbitrary irreducible character of $G$. Suppose that $\psi, \phi \in \operatorname{Irr}\left(\chi_{H}\right)$ and choose $\alpha \in \operatorname{Irr}\left(\psi_{K}\right)$ and $\beta \in \operatorname{Irr}\left(\phi_{K}\right)$. Since $\alpha$ and $\beta$ are constituents of $\chi_{K}$, they are $G$-conjugate by Clifford's theorem. Now the argument above and the condition $(*)$ shows that $\psi$ and $\phi$ have the same degree. Thus $(G, H) \in \Delta$ as claimed.

Corollary 6 Suppose that $H$ is a subgroup of $G$ and $H^{\prime} \triangleleft G$. If every $H$ conjugacy class lying in $H^{\prime}$ is mapped into itself under conjugation by $G$, then $(G, H) \in \Delta$.

Proof. A lemma of Brauer (see, [1] or [6, (6.32)]) applied to the character table of $H^{\prime}$ shows that the number of $G$-orbits (respectively $H$-orbits) on $\operatorname{Irr}\left(H^{\prime}\right)$ is equal to the number of $G$-orbits (respectively $H$-orbits) on the set of conjugacy classes of $H^{\prime}$. Thus the hypothesis shows that the number of $G$-orbits on $\operatorname{Irr}\left(H^{\prime}\right)$ is equal to the number of $H$-orbits on $\operatorname{Irr}\left(H^{\prime}\right)$. This implies that whenever $\alpha, \beta \in \operatorname{Irr}\left(H^{\prime}\right)$ are $G$-conjugate, then $\alpha^{x}=\beta$ for some $x \in H$, and hence $\alpha^{H}=\beta^{H}$. Hence the criterion of the theorem is satisfied and $(G, H) \in \Delta$.

Corollary 7 Suppose that $H \leq G$ with $H^{\prime} \triangleleft G$ and that, for each imprimitive irreducible character $\chi$ of $G$, all constituents of $\chi_{H}$ have the same degree. Then $(G, H) \in \Delta$.

Proof. By Theorem 5 we have to show that if $\alpha$ and $\beta$ are distinct irreducible characters of $H^{\prime}$ which are $G$-conjugate, then the degrees of the irreducible constituents of $\alpha^{H}$ and $\beta^{H}$ are the same. Since the characters are $G$-conjugate we can choose $\chi \in \operatorname{Irr}(G)$ such that $\alpha, \beta \in \operatorname{Irr}\left(\chi_{H^{\prime}}\right)$. The $G$-orbit for $\alpha$ has length $>1$, so $\chi$ is imprimitive (see $[6,(6.11)]$ ), and hence the degrees of the constituents of $\chi_{H}$ are all equal by hypothesis. These constituents include some constituents of both $\alpha^{H}$ and of $\beta^{H}$ by Frobenius reciprocity. Since all constituents of $\alpha^{H}$ have the same degree (and similarly for $\beta^{H}$ ), the result follows. 
Corollary 8 Suppose that $H \leq G$ with $H^{\prime} \triangleleft G$, and for each $x \in G$ there exists $\sigma \in A u t(H)$ such that $\sigma(u)=x^{-1} u x$ for all $u \in H^{\prime}$. Then $(G, H) \in \Delta$.

Proof. Suppose that $\alpha=\beta^{x}$ for $\alpha, \beta \in \operatorname{Irr}\left(H^{\prime}\right)$ and $x \in G$. Then $\alpha \circ \sigma=\beta$ where $\sigma \in \operatorname{Aut}(H)$ is chosen as above. Now a straightforward computation shows that $\beta^{H}=(\alpha \circ \sigma)^{H}=\alpha^{H} \circ \sigma$. For each $\psi \in \operatorname{Irr}(H)$ we have $\psi \circ \sigma \in \operatorname{Irr}(H)$ and $\psi(1)=(\psi \circ \sigma)(1)$. Thus the irreducible constituents of $\alpha^{H}$ and $\beta^{H}$ have the same degree as required.

In the special case where the degree homogeneous subgroup is a maximal subgroup, we can be more precise.

Theorem 9 Suppose that $H$ is a maximal subgroup of the group $G$ which is not normal in $G$, and put $K:=\operatorname{core}_{G}(H)$. Then $(G, H) \in \Delta$ if and only if the following conditions hold:

(i) $G / K$ is a solvable Frobenius group in which $H / K$ is an abelian Frobenius complement; and

(ii) if $M / K$ is the Frobenius kernel for $G / K$ then, for each $\alpha \in \operatorname{Irr}(K)$, either $I_{G}(\alpha) \leq M$ or $I_{G}(\alpha) \geq M$.

Proof. Suppose that properties (i) and (ii) hold. We shall show that $(G, H) \in \Delta$. Indeed, (i) shows that the normal subgroup $K$ of $G$ contains $H^{\prime}$ so it is sufficient to verify that condition $(*)$ of Theorem 5 holds. Suppose that $\alpha, \beta \in \operatorname{Irr}(K)$ are $G$-conjugate. Then the inertia subgroups for $\alpha$ and $\beta$ are conjugate in $G$. Thus, if $I_{G}(\alpha) \leq M \triangleleft G$, then $I_{G}(\beta) \leq M$, and hence $I_{H}(\alpha)=I_{H}(\beta)=K$. This implies that $\alpha^{H}$ and $\beta^{H}$ are irreducible. Since they have the same degree, $\left({ }^{*}\right)$ is satisfied in this case. On the other hand, $G=M H$ and so, if $I_{G}(\alpha) \geq M$, then every $G$-conjugate of $\alpha$ is also an $H$-conjugate. Thus $\alpha^{H}=\beta^{H}$ and so $\left(^{*}\right)$ is satisfied in this case as well.

Conversely, suppose that $(G, H) \in \Delta$. Then $H^{\prime} \triangleleft G$ and so $H^{\prime} \leq \operatorname{core}_{G}(H)=$ $K$. For each subgroup $L$ with $K \leq L \leq G$ we denote $L / K$ by $\bar{L}$. Then $\bar{H}$ is an abelian, maximal, core-free subgroup of $\bar{G}$, and hence $\bar{G}$ is a solvable, Frobenius group with $\bar{H}$ as a Frobenius complement (see [4] or [8, Theorem 13.4.6]). This proves (i).

Now consider (ii) and suppose that for some $\alpha \in \operatorname{Irr}(K)$ we have $I_{G}(\alpha) \nsupseteq M$. We have to show that $I_{G}(\alpha) \leq M$. Since $\bar{G}$ is solvable, and $\bar{H}$ is a Hall subgroup, we may replace $\alpha$ by a $G$-conjugate character and assume that $T:=I_{G}(\alpha)=N S$ where $N=T \cap M$ and $S=T \cap H$. By our assumption, $N \neq M$. For each $u \in \bar{N}$ we have $u \bar{S} u^{-1} \leq \bar{T} \cap u \bar{H} u^{-1}$. Since $\bar{H}$ is a Frobenius complement, the trivial intersection property shows that

$$
\left|\bigcup_{u \in \bar{N}} u \bar{S} u^{-1}\right|=|\bar{N}|(|\bar{S}|-1)+1=|\bar{T}|-(|\bar{N}|-1)
$$

Thus $\bar{T}=\bar{N} \cup \bigcup_{u \in \bar{N}} u \bar{S} u^{-1}$. Again by the property of a Frobenius complement, we conclude that $\bar{T} \cap v \bar{H} v^{-1}=1$ whenever $v \in \bar{M} \backslash \bar{N}$. Thus by our assumption that $M \neq N$ there exists $v$ such that $v^{-1} T v \cap H=K$. Since $I_{G}\left(\alpha^{v}\right)=v^{-1} T v$, 
we have $I_{H}\left(\alpha^{v}\right)=K$ and so $\left(\alpha^{v}\right)^{H}$ is irreducible (see [6, (6.11)]). Since $(G, H) \in$ $\Delta$, condition $\left(^{*}\right)$ of Theorem 5 holds, and so $\alpha^{H}$ must also be irreducible. This implies that $I_{H}(\alpha)=S=K$, and so $I_{G}(\alpha) \leq M$ as required.

Remark 10 The kernel $M / K$ of a solvable Frobenius group of the form described in the theorem above must be a minimal normal subgroup of $G / K$ and hence is elementary abelian. The abelian complement $H / K$ acts fixed point free on $M / K$ and so is cyclic (see, for example, [5, page 499]).

This gives another necessary condition for a degree homogeneous subgroup.

Corollary 11 Suppose that $(G, H) \in \Delta$ with $H \neq G$. Then, for each subgroup $L$ of $G$ in which $H$ is a maximal subgroup, $L^{\prime \prime} \leq H$ and $|L: H|$ is a prime power.

Proof. Lemma 1 shows that $(L, H) \in \Delta$. Since $H$ is maximal in $L$, either $H \triangleleft L$ and $|L: H|$ is a prime, or Theorem 9 applies. In the latter case the remark above shows that $L^{\prime \prime} \leq H$ and that $|L: H|$ is a prime power.

\section{Universal degree homogenous groups}

We shall call a finite group $H$ universally degree homogeneous if, whenever $H$ is embedded as a subgroup in a finite group $G$ with $H^{\prime} \triangleleft G$, then $(G, H) \in \Delta$. For example, abelian groups and perfect groups are universally degree homogeneous. The class of universal degree homogeneous groups can be characterized as follows.

We define an equivalence relation $\sim$ on $\operatorname{Irr}\left(H^{\prime}\right)$ by

$$
\alpha \sim \beta \text { if and only if } \alpha \circ \sigma=\beta \text { for some } \sigma \in \operatorname{Aut}\left(H^{\prime}\right)
$$

Theorem $12 A$ finite group $H$ is universally degree homogeneous if and only if $\alpha \sim \beta$ (for $\alpha, \beta \in \operatorname{Irr}\left(H^{\prime}\right)$ ) implies that the irreducible constituents of $\alpha^{H}$ and $\beta^{H}$ are all of the same degree.

Proof. Suppose that $\alpha \sim \beta$ (for $\alpha, \beta \in \operatorname{Irr}\left(H^{\prime}\right)$ ) implies that the irreducible constituents of $\alpha^{H}$ and $\beta^{H}$ are all of the same degree. If $H$ is embedded as a subgroup in $G$ with $H^{\prime} \triangleleft G$ and two irreducible characters of $\alpha$ and $\beta$ of $H^{\prime}$ are $G$-conjugate, then $\alpha \circ \sigma=\beta$ where $\sigma$ is the automorphism of $H^{\prime}$ induced by conjugation by some element of $G$, and therefore $\alpha \sim \beta$. Now $(G, H) \in \Delta$ by Theorem 5 . Thus $H$ is universally degree homogeneous.

Conversely, suppose that for some $\alpha \sim \beta$ (for $\alpha, \beta \in \operatorname{Irr}\left(H^{\prime}\right)$ ) the irreducible constituents of $\alpha^{H}$ and $\beta^{H}$ are not of the same degree. By hypothesis $\alpha \circ \sigma=$ $\beta$ for some $\sigma \in \operatorname{Aut}\left(H^{\prime}\right)$. Suppose that $\sigma$ has order $s$ and let $K$ be the semidirect product of $H^{\prime}$ by $\langle\sigma\rangle$. The permutational product construction of B.H. Neumann [7] of the amalgam $H$ and $K$ with common subgroup $H^{\prime}$ gives a permutation group $G$ of degree $|H| s$ in which $H$ and $K$ are embedded as subgroups with $H \cap K=H^{\prime}$ (in general, the construction does not give a 
unique $G$ ). Since $\alpha$ and $\beta$ are $K$-conjugate, they are also $G$-conjugate. Thus Theorem 5 shows that $H$ is not degree homogeneous in $G$ and hence $H$ is not universally degree homogeneous.

Example 13 Let $H$ be a group whose irreducible characters all have degrees 1 or $m$ for some $m>1$. Then $H$ is universally degree homogeneous. Indeed, if $\alpha \in \operatorname{Irr}\left(H^{\prime}\right)$ is not the trivial character, then Frobenius reciprocity shows that no irreducible constituent of $\alpha^{H}$ can have degree 1 and hence the criterion of Theorem 12 is satisfied. The class of groups whose characters have only two distinct degrees includes the dihedral groups, extraspecial p-groups (see, for example, [5, page 562]) and $A_{4}$. If $m=p$ is prime, then the class consists precisely of those groups $H$ such that: either $H$ has an abelian normal subgroup of index $p$; or $|H: Z(H)|=p^{3}$. For further information on this class of groups see [6, Chapter 10].

Example 14 Let $H$ be a Frobenius group with an abelian Frobenius complement. Then $H^{\prime}$ is the Frobenius kernel, and $\alpha^{H}$ is irreducible for every $\alpha \in$ $\operatorname{Irr}\left(H^{\prime}\right)$ with $\alpha \neq 1_{H^{\prime}}$ (see [6, (6.34)]). On the other hand, if $\alpha=1_{H^{\prime}}$, then $\alpha^{H}$ is equal to the sum of the characters of degree 1 for $H$ since $H / H^{\prime}$ is abelian. Thus $H$ is universally degree homogeneous by Theorem 12.

Example 15 If every automorphism of $H^{\prime}$ is induced by conjugation by an element of $H$, then $H$ is universally degree homogeneous. Indeed, in this case, if $\alpha, \beta \in \operatorname{Irr}\left(H^{\prime}\right)$ and $\alpha \sim \beta$ then $\alpha^{x}=\beta$ for some $x \in H$. Hence $\alpha^{H}=\beta^{H}$, and Theorem 12 applies. Similarly, if $S$ is a nonabelian simple group whose outer automorphism group is abelian, then any group $H$ satisfying $S \cong \operatorname{Inn}(S) \leq$ $H \leq \operatorname{Aut}(S)$ is universally degree homogeneous. Indeed, consider such an $H$ and suppose that $\alpha \sim \beta$ for some $\alpha, \beta \in \operatorname{Irr}\left(H^{\prime}\right)$. Since $H^{\prime} \cong S$, every automorphism of $H^{\prime}$ is induced by conjugation by some element in $A u t(S)$, and so $\alpha=\beta^{x}$ for some $x \in A u t(S)$. Since the outer automorphism group of $S$ is abelian, $H \triangleleft A u t(S)$ and so $\alpha^{H}=\beta^{x H}=\beta^{H x}$. Thus the irreducible constituents of $\alpha^{H}$ are images under $x$ of the irreducible constituents of $\beta^{H}$. Now Theorem 12 applies to show that $H$ is universally degree homogeneous. For example, all symmetric groups $S_{n}$ and subgroups of the projective general linear groups $P G L(2, p)$ (where $p>3$ is prime) which contain $P S L(2, p)$ are universally degree homogeneous.

\section{$4 \quad L$-classes of characters}

If $\psi$ is an irreducible character of degree $d$ in the group $H$ and $\lambda$ is a linear character of $H$ then $\psi \lambda$ is also an irreducible character of degree $d$. We define the $L$-class of $\psi$ to be $\{\psi \lambda \mid \lambda \in \operatorname{Lin}(H)\}$ and note that the $L$-classes partition $\operatorname{Irr}(H)$ into subsets of characters of the same degree.

Lemma $16 \mathcal{C} \subseteq \operatorname{Irr}(H)$ is an L-class of characters if and only if $\mathcal{C}=\operatorname{Irr}\left(\alpha^{H}\right)$ for some $\alpha \in \operatorname{Irr}\left(H^{\prime}\right)$. 
Proof. If $\alpha \in \operatorname{Irr}\left(H^{\prime}\right)$, then [6, Problem (6.2)] shows that $\alpha^{H}=f \sum_{i=1}^{t} \psi_{i}$ for some integer $f$ where the distinct characters $\psi_{1}, . ., \psi_{t}$ form an $L$-class in $\operatorname{Irr}(H)$. Thus $\operatorname{Irr}\left(\alpha^{H}\right)$ is an $L$-class. Conversely, if an $L$-class $\mathcal{C}$ contains an irreducible character $\psi$, say, of $H$, and we choose $\alpha \in \operatorname{Irr}\left(\psi_{H^{\prime}}\right)$, then Frobenius reciprocity shows that $\psi \in \operatorname{Irr}\left(\alpha^{H}\right)$. Since the $L$-classes partition $\operatorname{Irr}(H)$, this shows that $\mathcal{C}=\operatorname{Irr}\left(\alpha^{H}\right)$.

Let $H$ be a subgroup of $G$. Then we define $(G, H) \in \Delta^{*}$ if for each $\chi \in$ $\operatorname{Irr}(G)$ the irreducible constituents of $\chi_{H}$ are contained in a single $L$-class. Clearly $\Delta^{*} \subseteq \Delta$, and we have the following criterion.

Theorem 17 Let $H$ be a subgroup of $G$. The following conditions are equivalent:

(a) $(G, H) \in \Delta^{*}$;

(b) every $H$-conjugacy class in $H^{\prime}$ is also a $G$-conjugacy class;

(c) $H^{\prime} \triangleleft G$ and if $\alpha, \beta \in \operatorname{Irr}\left(H^{\prime}\right)$ are $G$-conjugate then they are also $H$ conjugate.

Proof. The lemma of Brauer quoted in the proof of Corollary 6 proves the equivalence of $(\mathrm{b})$ and $(\mathrm{c})$.

We next prove that (a) implies (c). The property $H^{\prime} \triangleleft G$ follows from Theorem 5 since $\Delta^{*} \subseteq \Delta$. Let $\alpha, \beta \in \operatorname{Irr}\left(H^{\prime}\right)$ be $G$-conjugate. Then $\alpha^{G}=\beta^{G}$. Choose $\chi \in \operatorname{Irr}\left(\alpha^{G}\right)$. Then Frobenius reciprocity shows that $\left[\chi_{H}, \alpha^{H}\right]=\left[\chi_{H}, \beta^{H}\right]=\left[\chi, \alpha^{G}\right]>0$. Now $(G, H) \in \Delta^{*}$ and Lemma 16 imply that $\operatorname{Irr}\left(\alpha^{H}\right)=\operatorname{Irr}\left(\beta^{H}\right)$, and indeed $\alpha^{H}=\beta^{H}$ since they have the same degree. On the other hand, $\left(\alpha^{H}\right)_{H^{\prime}}$ is a sum of characters which are $H$-conjugate to $\alpha$ whilst $\left(\beta^{H}\right)_{H^{\prime}}$ is a sum of characters which are $H$-conjugate to $\beta$. Thus $\alpha$ is $H$-conjugate to $\beta$.

Finally we prove that (c) implies (a). Assume that (c) holds. Let $\chi \in$ $\operatorname{Irr}(G)$ and let $\alpha \in \operatorname{Irr}\left(\chi_{H^{\prime}}\right)$. Since $\chi \in \operatorname{Irr}\left(\alpha^{G}\right)$, in proving (a) it is enough to show that $\operatorname{Irr}\left(\left(\alpha^{G}\right)_{H}\right) \subseteq \operatorname{Irr}\left(\alpha^{H}\right)$ (where the latter is a single $L$-class by Lemma 16). By hypothesis, the characters $\alpha=\alpha_{1}, \ldots, \alpha_{t}$ of $H^{\prime}$ which are $G$ conjugate to $\alpha$ are the same as those which are $H$-conjugate. Therefore there exist integers $e$ and $f$ such that

$$
\left(\alpha^{G}\right)_{H^{\prime}}=e\left(\alpha_{1}+\ldots+\alpha_{t}\right) \text { and }\left(\alpha^{H}\right)_{H^{\prime}}=f\left(\alpha_{1}+\ldots+\alpha_{t}\right)
$$

On the other hand, since $H^{\prime} \triangleleft G$, the induced characters $\alpha^{G}$ and $\alpha^{H}$ are both 0 outside of $H^{\prime}$. Thus $\left(\alpha^{G}\right)_{H}=(e / f) \alpha^{H}$, and so (a) follows.

In the following we shall use $\operatorname{Irr}\left(G, H^{\prime}\right)$ to denote the set of characters $\chi \in \operatorname{Irr}(G)$ for which $H^{\prime} \not \leq \operatorname{ker} \chi$.

Theorem 18 Suppose that $H$ is a subgroup of $G$ with $H^{\prime} \triangleleft G$. Then the following are equivalent:

(a) The sets $\operatorname{Irr}\left(\chi_{H}\right)\left(\chi \in \operatorname{Irr}\left(G, H^{\prime}\right)\right)$ are pairwise disjoint and each of these sets is a union of complete L-classes.

(b) For each $\chi \in \operatorname{Irr}\left(G, H^{\prime}\right)$ there exists $\alpha \in \operatorname{Irr}\left(H^{\prime}\right)$ and an integer $m$ such that $\alpha^{G}=m \chi$.

(c) For each $\chi \in \operatorname{Irr}\left(G, H^{\prime}\right)$ we have $\chi=0$ on $G \backslash H^{\prime}$. 
Proof. Assume that (a) holds; we shall prove (b). Let $\chi \in \operatorname{Irr}\left(G, H^{\prime}\right)$ and take $\alpha \in \operatorname{Irr}\left(\chi_{H^{\prime}}\right)$. Note that $\alpha \neq 1_{H^{\prime}}$ since $H^{\prime} \not \leq \operatorname{ker} \chi$, and Lemma 16 shows that $\mathcal{C}:=\operatorname{Irr}\left(\alpha^{H}\right)$ is an $L$-class with $\operatorname{Irr}\left(\chi_{H}\right) \cap \mathcal{C} \neq \emptyset$. . By Frobenius reciprocity $\chi \in \operatorname{Irr}\left(\alpha^{G}\right)$, so in order to prove (b) it is enough to show that $\operatorname{Irr}\left(\alpha^{G}\right)$ contains no other character. However, $\psi \in \operatorname{Irr}\left(\alpha^{G}\right)$ implies that $\alpha \in \operatorname{Irr}\left(\psi_{H^{\prime}}\right)$ (so $\left.\psi \in \operatorname{Irr}\left(G, H^{\prime}\right)\right)$ and $\operatorname{Irr}\left(\psi_{H}\right) \cap \mathcal{C} \neq \emptyset$. Thus (a) implies $\psi=\chi$ as required.

Clearly, (b) implies (c) because any character $\alpha^{G}$ induced from the normal subgroup $H^{\prime}$ is 0 outside of $H^{\prime}$.

Finally, assuming that (c) holds, we shall prove (a). Fix $\chi \in \operatorname{Irr}\left(G, H^{\prime}\right)$ and let $\psi \in \operatorname{Irr}(G)$. Then (c) shows that

$$
|G|[\chi, \psi]=\sum_{x \in H^{\prime}} \chi(x) \overline{\psi(x)}=\left|H^{\prime}\right|\left[\chi_{H^{\prime}}, \psi_{H^{\prime}}\right]
$$

and so $\operatorname{Irr}\left(\chi_{H^{\prime}}\right) \cap \operatorname{Irr}\left(\psi_{H^{\prime}}\right)=\emptyset$ whenever $\psi \neq \chi$. In particular, $\operatorname{Irr}\left(\chi_{H}\right)$ and $\operatorname{Irr}\left(\psi_{H}\right)$ are disjoint when $\psi \neq \chi$. On the other hand, Lemma 16 shows that if $\mathcal{C}$ is an $L$-class such that $\mathcal{C} \cap \operatorname{Irr}\left(\psi_{H}\right) \neq \emptyset$ then $\mathcal{C}=\operatorname{Irr}\left(\alpha^{H}\right)$ for some $\alpha \in \operatorname{Irr}\left(\psi_{H^{\prime}}\right)$. Thus $\mathcal{C} \cap \operatorname{Irr}\left(\chi_{H}\right) \neq \emptyset$ implies that $\mathcal{C} \cap \operatorname{Irr}\left(\psi_{H}\right)=\emptyset$ whenever $\psi \neq \chi$, and so $\operatorname{Irr}\left(\chi_{H}\right)$ consists of complete $L$-classes. This proves that (c) implies (a).

Corollary 19 Whenever $H$ and $G$ satisfy the (equivalent) conditions of Theorem 18 and $G \neq H^{\prime}$ then:

(i) $H^{\prime}$ is solvable and p-nilpotent for all primes $p|| G: H^{\prime} \mid$;

(ii) for each $x \in G \backslash H^{\prime}$ and each $u \in H^{\prime}, x$ is conjugate to $x u$ in $G$; and

(iii) if $H^{\prime}$ is a Hall subgroup of $G$, then $G$ is a Frobenius group with Frobenius kernel $H^{\prime}$.

Proof. (i) Let $\chi \in \operatorname{Irr}(G)$ with $H^{\prime} \not \leq$ ker $\chi$. Then by part (b) we have $m \chi=\alpha^{G}$ for some integer $m$ and some $\alpha \in \operatorname{Irr}\left(H^{\prime}\right)$. By Frobenius reciprocity and Clifford's theorem, $\chi_{H^{\prime}}=m\left(\alpha_{1}+\ldots,+\alpha_{s}\right)$ where $\alpha_{1}, \ldots, \alpha_{s}$ are the distinct $G$-conjugates of $\alpha$. Thus $m \mid \chi(1)$ and $m \chi(1)=\alpha^{G}(1)=\alpha(1)\left|G: H^{\prime}\right|$. Let $p$ be a prime which divides $\left|G: H^{\prime}\right|$. Then $p \mid \chi(1)$ whenever $H^{\prime} \not \leq \operatorname{ker} \chi$. Now it is proved in [2, page 21] (for a general finite group $G$ ) that if $X$ is the set of all $\chi \in \operatorname{Irr}(G)$ such that $\chi(1)>1$ and $p \nmid \chi(1)$, then $G\left(p^{\prime}\right):=\bigcap_{\chi \in X} \operatorname{ker} \chi$ is a solvable $p$-nilpotent subgroup of $G$. In our case $G\left(p^{\prime}\right) \geq H^{\prime}$, and so $H^{\prime}$ is solvable and $p$-nilpotent for all $p|| G: H^{\prime} \mid$.

(ii) It is enough to show that $\chi(x)=\chi(x u)$ for all $\chi \in \operatorname{Irr}(G)$. If $H^{\prime} \not \leq \operatorname{ker} \chi$, then this follows from part (c) of the theorem (both sides are 0 ). On the other hand, if $H^{\prime} \leq \operatorname{ker} \chi$, then a representation affording $\chi$ maps $u$ onto the identity and so again we have $\chi(x)=\chi(x u)$ as required.

(iii) If $H^{\prime}$ is a Hall subgroup then by the Schur-Zassenhaus theorem $H^{\prime}$ has a complement, $K$ say, in $G$. Now (ii) shows that every $x \in G \backslash H^{\prime}$ is conjugate in $G$ to some element of $K$. This is sufficient to show that $G$ is a Frobenius group with kernel $H^{\prime}$ (see, for example, [6, Problem (7.1)]) 
Acknowledgement The authors wish to thank an anonymous referee for making several useful suggestions and, in particular, pointing out an omission in an earlier version of Theorem 18.

\section{References}

[1] R. Brauer, On connections between ordinary and modular characters of groups of finite order. Ann. of Math. (2) 42 (1941), 926-935.

[2] Ya. G. Berkovich and E.M. Zhmud', Characters of Finite Groups II. Vol. 181, Math. Monographs, Amer. Math. Soc., Rhode Island, 1999.

[3] The GAP Group, GAP-Groups, Algorithms, and Programming. Version 4.3 (2002) (http://www.gap-system.org).

[4] I.N. Herstein, A remark on finite groups. Proc. Amer. Math. Soc. 9 (1958), $255-257$.

[5] B. Huppert, Endliche Gruppen I. Springer-Verlag, Berlin, 1967.

[6] I.M. Isaacs, Character Theory of Finite Groups. Academic Press, New York, 1976.

[7] B.H. Neumann, Permutational products of groups. J. Austral. Math. Soc. 1 (1959/60), 299-310.

[8] W.R. Scott, Group Theory. Prentice-Hall, New Jersey, 1964.

John D. Dixon

School of Mathematics and Statistics

Carleton University

Ottawa ON K1S 5B6

Canada

email: jdixon@math.carleton.ca

A. Rahnamai Barghi

Institute for Advanced Studies in Basic Sciences

P.O. Box 45195-159

Zanjan, Iran

email: rahnama@iasbs.ac.ir 